\title{
LASIK em programa de treinamento médico: complicações relacionadas a microcerátomos
}

\author{
LASIK in a residency program:microkeratome-related complications
}

\author{
Eliana Doming'ues Gonçalves* \\ Mauro Campos* \\ Helena Tanaka*
}

\footnotetext{
Trabalho realizado no Setor de Cirurgia Refrativa, Departamento de Oftalmologia, Universidade Federal de São Paulo - UNIFESP.

* Médicos do Setor de Cirurgia Refrativa do Departamento de Oftalmologia da UNIFESP.

Endereço para correspondência: Praça Miguel Couto, 63 - ap. 92 - Santos (SP) CEP 11040-030

E-mail: elianadg@ig.com.br

Recebido para publicação em 10.10.2002

Versão revisada recebida em 19.02.2003

Aprovação em 07.04.2003
}

\begin{tabular}{|l|}
\hline RESUMO \\
\hline Objetivo: Avaliar as complicações da ceratomileuse assistida por excimer \\
laser in situ - LASIK - relacionadas ao microcerátomo em um centro de \\
treinamento médico. Métodos: O total de 1611 olhos submetidos a cerato- \\
mileuse assistida por excimer laser in situ foram estudados retrospectiva- \\
mente no período de janeiro de 1998 a junho de 2000 no setor de cirurgia \\
refrativa do Departamento de Oftalmologia da UNIFESP-EPM. Resulta- \\
dos: As complicações relacionadas com o microcerátomo na ceratomileuse \\
assistida por excimer laser in situ foram 26 (1,61\%), das quais 9(34,61\%) \\
foram de lamelas incompletas, 9 (34,61\%) finas, 2 (7,69\%) lamelas sem \\
pedículo, 5 (19,23\%) irregulares e 1 (3,84\%) perfurada. Conclusão: A \\
prevalência de complicações com microcerátomo em centro de treinamento \\
médico foi baixa e o treinamento de residentes e estagiários mostrou ser \\
uma prática viável.
\end{tabular}

Descritores: Ceratomileuse assistida por excimer laser in situ/efeitos adversos; Cirurgia a laser; Córnea/cirurgia; Internato e residência; Complicações intra-operatórias

\section{INTRODUÇÃO}

A técnica do LASIK (Laser in situ keratomileusis) está associada a idéia de recuperação rápida da acuidade visual e conforto no pós-operatório, por oftalmologistas e pacientes de cirurgia refrativa ${ }^{(1-3)}$. A confecção da lamela corneana pediculada, apesar de ser uma técnica segura e facilmente reprodutível, apresenta percentual de complicações em torno de 0,4\% a 10\%, e o manuseio dos microcerátomos está associado a uma curva de aprendizado ${ }^{(2-5)}$. As complicações geralmente estão relacionadas ao tempo de sucção ou ao microcerátomo e são descritas as eventuais perfurações corneanas, lamelas incompletas, irregulares, finas, sem pedículo ("free cap") e perfurada ("buttonhole") ${ }^{(1-14)}$.

Estudam-se os fatores predisponentes para cada uma delas como ceratometria prévia, irregularidades corneanas, diâmetro corneano, sincronismo entre os motores de avanço e vibração da lâmina do microcerátomo, sucção e PO (pressão intra-ocular) inadequadas, assim como a melhor conduta para cada caso $^{(2)}$.

Estas complicações podem evoluir com astigmatismos irregulares, cicatrizes estromais, "haze" e epitelização da interface e apresentar sintomas como ofuscamento ("glare") e halos, diplopias monoculares e perda de linhas de visão, piorando o prognóstico e dificultando um segundo procedimento ${ }^{(2,4,8,10)}$. A perda significativa de linha de visão (mais de 2 linhas) ocorre em torno de $4 \%{ }^{(11)}$.

Este estudo avalia as complicações per-operatórias relacionadas à confecção da lamela no LASIK em um serviço universitário, no período de janeiro de 1998 a junho de 2000, quanto à prevalência, curva de aprendizado e possíveis fatores predisponentes. 


\section{MÉTODOS}

\section{Seleção dos pacientes}

Foi realizada uma análise de revisão de 1611 cirurgias com a técnica do LASIK no período de janeiro de 1998 a junho de 2000, no Setor de Cirurgia Refrativa do Departamento de Oftalmologia da Universidade Federal de São Paulo - UNIFESP. As fichas dos pacientes foram acessadas por meio do livro de registro de cirurgias do serviço, de onde foram anotados: sexo, idade, profissão, dados pré-operatórios do olho operado, laser utilizado, número de reoperações, tempo de pósoperatório, complicações relacionadas ao corte do microcerátomo (lamelas incompletas, finas, irregulares, sem pedículo, perfuradas) e a experiência do cirurgião.

\section{Exame oftalmológico}

No pré-operatório foram realizados exames de acuidade visual com e sem correção, refração estática sob cicloplegia, topografia corneana, paquimetria ultra-sônica, medida do tamanho da pupila mesópica e escotópica, biomicroscopia, tonometria de aplanação e oftalmoscopia binocular indireta. Estes exames foram repetidos com um mês, três, seis meses e a cada ano de pós-operatório.

\section{Técnica cirúrgica}

Os cirurgiões foram residentes do terceiro ano e estagiários em treinamento em cirurgia refrativa monitorados por um cirurgião experiente.

Foram realizadas 1611 cirurgias (LASIK), todas unilaterais, com microcerátomo da Moria C-B ${ }^{\circledR}$ com avanço elétrico, manual ou com o microcerátomo Automated Corneal Shaper ACS - Chiron ${ }^{\circledR}$, no laser Summit SVS Apex plus ${ }^{\circledR}$ ou no laser Aesculap-Meditec-Mel 70 ${ }^{\circledR}$.

As cirurgias foram realizadas após a instilação de colírio anestésico, assepsia da pele, proteção da margem palpebral com adesivos, colocação de blefarostato, marcação corneana com violeta de genciana, posicionamento do anel de sucção concêntrico à pupila, encaixe da cabeça do microcerátomo após aferição da PO (pressão intra-ocular) adequada, ablação do estroma com excimer laser, limpeza da interface com solução salina balanceada, reposicionamento e secagem da margem da lamela com esponja de celulose.

\section{RESULTADOS}

Foram analisados os dados de 1.611 olhos, dos quais 26 olhos (1,6\%) apresentaram complicações durante a confecção da lamela, sendo 14 (53,8\%) de indivíduos do sexo masculino, com idades variando entre 22 a 52 anos, média de 30,3 anos; mediana de 28 anos.

O período de evolução foi de cinco meses a quatro anos.

Predominaram os pacientes de cor branca 24 olhos (92,3\%) e o olho direito com 16 olhos (59,2\%). As complicações ocor- reram em 18 olhos $(69,2 \%)$ submetidos ao microcerátomo Automated corneal Shaper /ACS-Chiron ${ }^{\circledR}$ com platô de 160 micra e 1 (3,8\%) com platô de 130 micra, 6 (23,0\%) no Moria C$\mathrm{B}^{\circledR}$ elétrico e 1 (3,8\%) no Moria C-B ${ }^{\circledR}$ manual, sendo 21 (80,7\%) no laser Summit SVS Apex plus ${ }^{\circledR}$.

Todas as cirurgias foram feitas por estagiários ou residentes do terceiro ano. O excimer laser não foi aplicado em 12 $(46,1 \%)$ cirurgias devido a confecção de lamelas inadequadas, sendo que 9 (34,6\%) foram reoperadas no período de 2,5 meses a 7 meses (média de 4,5 meses), 3 (11,5\%) apresentaram complicações nas reoperações e $3(11,5 \%)$ não reoperaram. Veja Tabelas 1 e 2.

\section{DISCUSSÃO}

O Departamento de Oftalmologia da UNIFESP, setor de Cirurgia Refrativa apresentou níveis baixos de prevalência de complicações da lamela no LASIK (1,6\%) em comparação a literatura (0,4\% a $10 \%)$, apesar das cirurgias serem feitas por cirurgiões em treinamento (estagiários e residentes do terceiro ano). Segundo Koch, a monitorização de um cirurgião experiente (orientador) é importante na redução do número de complicação no período inicial do treinamento médico ${ }^{(11)}$.

A predominância de lamelas incompletas 9 (34,6\%), pode estar relacionada a inexperiência do cirurgião, falha ou obstáculos no sistema de engrenagens do microcerátomo. Na tabela 1 observa-se que uma, no total de duas lamelas sem pedículo, apresentava ceratometria inferior a 41,25; e o único com lamela perfurada $1(3,8 \%)$ apresentou ceratometria baixa, contrariando a expectativa de predomínio em córneas mais curvas, apesar de ser conhecida sua etiologia multifatorial como diâmetro corneano, perda da pressão intra-ocular, elasticidade corneana, irregularidades na lâmina, sendo sua incidência na literatura de 0,3 a $2,6 \%{ }^{(2,10)}$.

O laser foi aplicado em 14 (53,8\%) olhos dos 9 (34,6\%) reoperados, 3 (33,3\%) apresentaram segunda complicação na reoperação e $1(11,1 \%)$ terceira complicação na segunda reoperação. Nos casos reoperados 1 (11,1\%) perdeu 2 linhas (paciente 7, veja tabelas 1 e 2).

Oito (30,7\%) olhos perderam linhas de visão, sendo que 5 $(62,5 \%)$ destes a aplicação de laser foi realizada no mesmo tempo cirúrgico da intercorrência da confecção da lamela corneana. Quatro (15,3\%) olhos perderam 2 linhas de visão sendo 2 olhos (50\%) devido a lamelas irregulares e 2 olhos (50\%) incompletas, em 3 (75\%) destes foi aplicado laser.

As piores complicações no sentido de perda de linha de visão foram as lamelas incompletas e finas, 3 (37,5\%) e 2 (25\%) respectivamente.

Este estudo mostrou que 18 (69,2\%) olhos apesar das complicações não perderam linhas de visão, e apenas 4 $(15,3 \%)$ perderam 2 linhas e nenhum mais que 2 linhas, sendo bom o prognóstico e a evolução dos casos. ${ }^{(2-4,7)} \mathrm{O}$ tempo de pós-operatório necessário para o segundo procedimento cirúrgico é descrito na literatura de 3 meses podendo ser menor 


\begin{tabular}{|c|c|c|c|c|c|}
\hline \multirow{2}{*}{$\begin{array}{l}\mathrm{N}^{\circ} \\
\text { pac. }\end{array}$} & \multirow{2}{*}{$\begin{array}{l}\text { Tipo de } \\
\text { complicação }\end{array}$} & \multicolumn{3}{|c|}{ Exame pré-operatório } & \multirow{2}{*}{$\begin{array}{l}\text { AV final s/c; c/c; (ceratometria média) } \\
\text { (pós-operatória) }\end{array}$} \\
\hline & & $\overline{A V ~ c / c}$ & Rx estática & Ceratometria (média) & \\
\hline 1 & $\begin{array}{l}\text { irregular* }^{*} \\
\text { (epitelização) }\end{array}$ & $20 / 25 p$ & $(-1,00 /-1,50 \times 165)$ & $43,50 \times 178 / 44,37 \times 88 \quad(43,9)$ & $20 / 60 ; 20 / 40(+1,25) ;(34,6)$ \\
\hline 2 & incompleta & $20 / 20$ & $(-4,25 /-0,75 \times 180)$ & $41,37 \times 178 / 42,50 \times 88(41,4)$ & 20/ 20; $(39,3)$ \\
\hline 3 & incompleta & $20 / 20 p$ & $(-2,25 /-1,75 \times 180)$ & $44,87 \times 6 / 46,62 \times 96(45,7)$ & 20/20p; $(43,2)$ \\
\hline 4 & fina & $20 / 20$ & $(-5,25 /-1,50 \times 20)$ & $42,87 \times 6 / 44,25 \times 93(43,5)$ & 20/20p; $(39,6)$ \\
\hline 50 & incompleta & $20 / 20$ & $(-3,00 /-2,00 \times 180)$ & $43,00 \times 3 / 45,25 \times 93 \quad(44,1)$ & 20/25p; $(43,8)$ \\
\hline 6 & incompleta & $20 / / 25$ & $(-5,75 /-2,75 \times 165)$ & $40,50 \times 155 / 43,50 \times 65(42,0)$ & 20/40p; $(37,3)$ \\
\hline 70 & incompleta & 20/15 & $(-2,00 /-1,50 \times 180)$ & $44,12 \times 178 / 43,12 \times 86 \quad(42,1)$ & 20/25; $(39,7)$ \\
\hline 8 & incompleta & $20 / 20$ & $(-4,25 /-0,75 \times 180)$ & $40,70 \times 177 / 42,00 \times 87(41,1)$ & $20 / 15 p ;(40,9)$ \\
\hline 9 & fina & $20 / 40$ & $(-8,50 /-1,75 \times 155)$ & $40,62 \times 164 / 42,25 \times 74(41,4)$ & 20/50; 20/30p $(-1,50) ;(36,4)$ \\
\hline 100 & fina $^{*}$ & $20 / 20 p$ & $(-1,50 /-2,50 \times 175)$ & $43,94 \times 82 / 42,34 \times 172(43,1)$ & 20/20; (42,9) *(incompleto) \\
\hline 11 & fina & $20 / 20$ & $(-7,50 /-0,75 \times 90)$ & $43,83 \times 16 / 43,71 \times 106(43,7)$ & $20 / 60 ; 20 / 25(-1,75 /-0,50 \times 90) ;(39,4)$ \\
\hline 12 & fina & $20 / 20 p$ & $(+2,00 /-2,50 \times 180)$ & $44,12 \times 175 / 46,00 \times 85(44,0)$ & $20 / 50 ; 20 / 15(+1,75 /-3,00 \times 5) ;(43,7)$ \\
\hline 13 & incompleta & $20 / 25$ & $(-6,50 /-1,75 \times 95)$ & $45,50 \times 14 / 45,12 \times 104(45,3)$ & $20 / 25 ;(44,7)$ \\
\hline 14 & fina & $20 / 20$ & $(-7,00 /-1,50 \times 180)$ & $44,12 \times 2 / 46,25 \times 92(45,1)$ & 20/20; $(39,3)$ \\
\hline 150 & fina & $20 / 15 p$ & $(-5,00 /-0,50 \times 40)$ & $41,87 \times 171 / 42,50 \times 81 \quad(42,1)$ & 20/20p; $(40,8)$ \\
\hline $16 a$ & sem pedículo* & $20 / 20$ & $(-2,00 /-2,50 \times 5)$ & $42,75 \times 4 / 45,62 \times 91(44,1)$ & 20/20; (42,4); *(incompleto) \\
\hline 170 & irregular & $20 / 20$ & $(-4,00 /-3,75 \times 180)$ & $41,37 \times 180 / 44,50 \times 90(42,9)$ & 20/20p; $(36,9)$ \\
\hline 18 & irregular & $20 / 20$ & $(-7,00)$ & $41,87 \times 152 / 42,13 \times 62(42,0)$ & 20/25; $(35,1)$ \\
\hline 19 & sem pedículo & $20 / 20 p$ & $(-2,75 /-3,75 \times 180)$ & $37,62 \times 176 / 40,25 \times 186(38,9)$ & 20/60p; 20/40 (-0,25/-2,50x10); $(36,2)$ \\
\hline 20 & fina & $20 / 20$ & $(-3,50 /-1,00 \times 160)$ & $41,37 \times 171 / 42,87 \times 81 \quad(42,1)$ & $20 / 20 ;(38,2)$ \\
\hline 210 & incompleta & $20 / 20$ & $(-4,50 /-0,50 \times 180)$ & $44,70 \times 91 / 43,80 \times 1 \quad(44,2)$ & $20 / 20 ;(44,0)$ \\
\hline 220 & perfurada & $20 / 20 p$ & $(-4,25 /-3,50 \times 10)$ & $40,87 \times 137 / 41,87 \times 87 \quad(41,3)$ & 20/20; $(40,6)$ \\
\hline 23 & fina & $20 / 20 p$ & $(-6,25 /-1,75 \times 180$ & $43,37 \times 177 / 45,62 \times 87 \quad(44,4)$ & 20/20p; $(40,6)$ \\
\hline 24 & irregular & $20 / 30$ & $(-4,50 /-1,50 \times 95)$ & $43,66 \times 0 / 42,93 \times 90(43,2)$ & 20/30p; $(39,7)$ \\
\hline 25 & incompleta* (dupla) & $20 / 20 p$ & $(+3,75)$ & $42,75 \times 177 / 43,12 \times 87 \quad(42,9)$ & $20 / 150 ; 20 / 20 p(+3,50) ;(43,1)$ \\
\hline 260 & irregular & $20 / 20$ & $(+5,25)$ & $41,70 \times 120 / 41,40 \times 30(41,5)$ & $20 / 50 ; 20 / 20(+3,25 /-1,00 \times 85) ;(42,7)$ \\
\hline
\end{tabular}

\begin{tabular}{|c|c|c|c|}
\hline $\begin{array}{l}\mathrm{N}^{\circ} \\
\text { pac. }\end{array}$ & $\begin{array}{l}\text { Tempo } \\
\text { (meses) }\end{array}$ & Complicações & $\begin{array}{l}\text { Perda de linha } \\
\text { de visão }\end{array}$ \\
\hline 5 & 3 & ausente & 1 \\
\hline 7 & 3 & ausente & 2 \\
\hline 10 & 7 & lamela irregular & 0 \\
\hline 15 & 7 & ausente & 1 \\
\hline 16 & 2,5 & ausente & 0 \\
\hline 17 & 3 & lamela incompleta & 0 \\
\hline 21 & 4,5 & ausente & 0 \\
\hline 22 & 4 & ausente & 0 \\
\hline \multirow[t]{2}{*}{26} & $5\left(1^{\circ}\right.$ reop $\left.^{\star}\right)$ & lamela irregular & 0 \\
\hline & $5\left(2^{\circ}\right.$ reop $\left.^{*}\right)$ & lamela dupla & 0 \\
\hline
\end{tabular}

no caso de lamelas incompletas. Neste estudo a média foi de 4,5 meses.

A aplicação ou não do laser após uma intercorrência na confecção da lamela deve ser avaliada. Sugere-se abortar a cirurgia. Devido a esta possibilidade sugerimos a orientação do paciente no pré-operatório quanto às complicações pósoperatórias e per-operatórias e quanto um possível segundo tempo cirúrgico.
A prevenção das complicações utilizando um "check-list" é importante, assim como a explicação de cada passo cirúrgico e desconforto relacionados ao anel de sucção e ao microcerátomo.

\section{CONCLUSÃO}

As complicações na confecção da lamela corneana apresentaram prevalência baixa e o treinamento de residentes e estagiários em cirurgia refrativa mostrou ser uma prática viável.

\section{ABSTRACT}

Purpose: To evaluate microkeratome complications related to laser in situ keratomileusis - LASIK - in a residency program. Methods: A total of 1,611 eyes were retrospectively evaluated after being submitted to laser in situ keratomileusis - LASIK surgery from January 1998 to June 2000 in the Refractive Surgery Clinic. Results: There were 26 (1.61\%) complications on performing the flap with the microkeratome and, described as 9 (34.61\%) incomplete flap, 9 (34.61\%) thin, 2 (7.69\%) free cap, 5 (19.23\%) irregular, 1 (3.84\%) buttonhole. Conclusion: The prevalence of microkeratome complications in a residency 
program was low and the training of residents and trainees in refractive surgery has shown to be a viable practice.

Keywords: Keratomileusis laser in situ/adverse effects; Laser surgery; Cornea/surgery; Internship and residency; Intraoperative complications

\section{REFERÊNCIAS}

1. Cameron BD, Saffra NA, Strominger MB. Laser in situ keratomileusis-induced optic neuropathy [commented on Opthalmology 2002;109:818, discussion p. 818-0]. Ophthalmology 2001;108:660-5.

2. Gimbel HV, Penno EE, Van Westenbrugge JA, Ferensowicz M, Furlong MT. Incidence and management of intraoperative and early postoperative complications in 1000 consecutive laser in situ keratomileusis cases [commented on Opthalmology 1999;106:1455-7]. Ophthalmology 1998;105:1839-47, discussion p.1847-8.

3. Gimbel HV, Basti S, Kaye GB, Ferensowicz M. Experience during the learning curve of laser in situ keratomileusis [commented on J Cataract Refract Surg 1996;22:513-4]. J Cataract Refract Surg 1996;22:542-50.

4. Tham VMB, Maloney RK. Microkeratome Complications of Laser In situ Keratomileusis. Ophthalmology 2000;107:920-4.
5. Wilson SE. LASIK: Management of common complications, Laser in situ keratomileusis. Cornea 1998;17:459-67.

6. Davidorf JM, Zaldivar R, Oscherow S. Results and complications of laser in situ keratomileusis by experience surgeons. J Refract Surg 1998;14:114-22.

7. Walker MB, Wilson SE. Lower intraoperative flap complication rate with the Hansatome Microkeratome Compared to the Automated Corneal Shaper [commented on J Refract Surg 2000;16:380]. J Refract Surg 2000;16:79-82.

8. Rao SK, Padmanabhan P, Sitalakshmi G, Rajagopal R, Lam DS. Timing of retreatment after a partial flap during laser in keratomileusis. J Caract Refract Surg 1999;25:1424-5.

9. Lam DS, Cheng AC, Leung AT. Lasik complications [commented on Ophthalmology 1998;105:1839-47, discussion p.1847-8]. Ophthalmology 1999; 106:1455-8.

10. Leung AT, Rao SK, Cheng AC, Yu EW, Fan DS, Lam DS. Pathogenesis and management of laser in situ Keratomileusis flap buttonhole [commented on J Cataract Refract Surg 2000;26:1270-1]. J Cataract Refract Surg 2000; 26:358-62.

11. Koch DD. Outcomes of initial PRK and Lasik procedures [commented on J Cataract Refract Surg 2000;26:497-509]. J Cataract Refract Surg 2000;469-70.

12. Pereira T, Forseto AS, Nose W. Complicações per e pós-operatórias em 1000 olhos submetidos a Lasik. Arq Bras Oftalmol 2001;64:499-506.

13. Veneziano PO, Bernardini MC. LASIK: Complicações com o uso de microcerátomos Automated Corneal Shaper e Hansatome - 1513 casos. Rev Bras Oftalmol 2001;60:782-7.

14. Pallikaris IG, Katsanevaki VJ, Panagopoulou SI. Laser in situ keratomileusis intraoperative complications using one type of microkeratome. Ophthalmology 2002;109:57-63.

\section{CONGRESSO INTERNACIONAL DA SOCIEDADE BRASILIENSE \\ dE OfTALMOLOGIA}

\section{7 e 8 de Maio de 2004}

\section{BRASÍLIA - DF}

INFORMAÇÕES: Tel/Fax: (61) 248-4030/8116-3304

com Dr. Hilton Medeiros ou

Tel/Fax: (61) 248-4030/346-6971

com Ana Paula 